\title{
Teorías más Utilizadas en la Negociación de Precios Colaborativos entre Empresas de la Cadena de Suministros
}

\author{
Alberto Ayaviri-Panozo(1) y Patricio E. Ramírez-Correa(2)* \\ (1) Facultad de Ciencia y Tecnología, Universidad San Francisco Xavier de Chuquisaca, Regimiento Campos 180, \\ Sucre, Bolivia (e-mail: ayaviri.alberto@usfx.bo). \\ (2) Escuela de Ingeniería, Universidad Católica del Norte, Larrondo 1281, Coquimbo, Chile. \\ (e-mail: patricio.ramirez@ucn.cl).
}

* Autor a quien debe ser dirigida la correspondencia.

Recibido Feb. 21, 2019; Aceptado Abr. 15, 2019; Versión final May. 27, 2019, Publicado Dic. 2019

\begin{abstract}
Resumen
Se identifican las teóricas más utilizadas en la investigación sobre negociación de precios colaborativos entre empresas de la cadena de suministros. La literatura seleccionada de la base de datos de Web of Science del período 2014-2018 se utilizó bajo el modelo de enfoque meta-analítico para distinguir las teorías más utilizadas en el tema estudiado. El análisis de 7680 artículos seleccionados se realizó con la ayuda del Software Vosviewer, identificándose las revistas más relevantes y los autores más citados. Mediante un análisis de co-citación se seleccionaron los autores relacionados con los enfoques teóricos, donde se identificaron también los más citados. Se concluye que la teoría prospectiva y la teoría de confirmación de expectativas son las más citadas. Las teorías de costos de transacción y de recursos y capacidades son relevantes para una gestión de negociación de precios colaborativos entre empresas de la cadena de suministros.
\end{abstract}

Palabras clave: enfoque meta-analítico; teorías; precios colaborativos; cadena de suministros; negociación

\section{The most Used Theories in the Collaborative Price Negotiation between Companies of the Supply Chain}

\begin{abstract}
The most commonly used theorists in the research on collaborative price negotiation between companies in the supply chain have been identified. The literature selected from the Web of Science database for the period 2014-2018 was used under the meta-analytic approach model to distinguish the most commonly used theories in the subject studied. The analysis of 7680 selected articles was carried out with the help of the Vosviewer Software, identifying the most relevant journals and the most cited authors. By means of a co-citation analysis the authors related to the theoretical approaches were selected, identifying again the most cited authors. It is concluded that the prospective theory and the confirmation theory of expectations are the most cited ones. The theories of transaction costs and resources and capacities are relevant for a negotiation management of collaborative prices between companies in the supply chain.
\end{abstract}

Keywords: meta-analytical approach; theories; collaborative prices; supply chain; negotiation 


\section{INTRODUCCIÓN}

En busca de la competitividad los directivos empresariales van transformando sus organizaciones de manera estratégica en base a sus recursos, el desarrollo de sus capacidades empresariales y buscan conformar cadenas de suministro para desarrollar sus procesos productivos. Mentzer et al. (2001) menciona que las industrias también dependen de los flujos de información, materiales, recursos económicos, mano de obra y bienes de capital. Forrester (1958 citado en Mentzer et al.,2001) manifestó que la "forma en que estos cinco sistemas de flujo se interconectan para ampliarse y causar cambios, constituirán una base que permitirá anticipar los efectos de las decisiones, las políticas, las formas de organizarse y las opciones de inversión". (p.1). De esta manera se introduce la teoría de la gestión de distribución de naturaleza integrada y organizacionalmente relacionada, dando inicio a la cadena de suministros. A lo largo del tiempo su definición fue ampliándose desde un proceso único en las diferentes áreas de la organización como algo que existe, pasando por la Gestión de la Cadena de Suministro como una coordinación sistémica a lo largo de la Cadena de Suministro (Mentzer et al., 2001). Actualmente las nuevas tendencias hablan de Gestión de la Cadena de Suministros Verde (Sarkis, Zhu, y Lai, 2011), donde se incorpora un comportamiento de cuidado ambiental.

La literatura manifiesta que la cadena de suministros es un conjunto de empresas que participan en sus diferentes procesos y actividades para generar valor (La Londe y Masters,1994 y Mentzer et al., 2001). Es decir, las empresas que interactúan con sus cinco sistemas propuestos por Forrester generaran valor a través de un producto final. A partir de estas proposiciones Mentzer et al. (2001) manifiesta que los individuos participan directamente en los flujos de productos, servicios, finanzas e información, que fluyen de un proveedor a un cliente y viceversa mediante una cadena de suministros directa, extendida y final. La cadena de suministro representa una red de suministro de material, su transformación en un producto terminado y la distribución de estos productos a los clientes finales generando calidad en los procesos (Salas-Navarro, Meza, Obredor-Baldovino y Mercado-Caruso, 2019). Por lo tanto, una cadena de suministros tiene actividades operativas que necesariamente deben realizarse para trasladar un producto desde una fuente hacia un cliente, para realizar cada una de estas actividades es necesario un proceso de gestión, surgiendo la Gestión de la Cadena de Suministros (SCM). La Londe y Masters (1994) han manifestado que las empresas realizan acuerdos de entendimiento de largo plazo y trabajan intensamente para alcanzar altos niveles de confianza y compromiso, para lograr una mejora en los servicios y la reducción de costos para los miembros en todos los niveles de la cadena de suministros. Donde la cadena como un todo se vuelve más competitiva en comparación con empresas de la industria que trabajan individualmente.

De las diversas definiciones que se plantearon para la SCM, Mentzer et al. (2001) indica que estas se pueden clasificar en tres categorías: una filosofía de gestión, la implementación de una filosofía de gestión y un conjunto de procesos de gestión. Una propuesta de SCM que considera las diversas definiciones tomando en cuenta que involucra varias empresas, muchas actividades empresariales y la coordinación de estas actividades, fue planteada por Mentzer et al. (2001) definiendo la SCM como la coordinación estratégica de funciones empresariales de una empresa en particular y en la cadena de suministros, para mejorar el rendimiento en el largo plazo de las empresas y de la cadena de suministros. La gestión efectiva de la Cadena de Suministro permite una mejor prestación de servicio al cliente y de la cadena de valor, a través de la gestión de los flujos de información, de productos y gestión económica (Sanchis, Poler y Ortiz, 2009)

El desarrollo de cadenas de suministro en las industrias ha permitido que la negociación de precios ya no vea el precio como una base de distribución entre socios comerciales, sino como una oportunidad para maximizar el margen bruto y el beneficio (Voeth y Herbst, 2006), logrando de esta manera beneficios mutuos para los integrantes de la cadena de suministros. Johansson y Andersson (2012) identificaron la conveniencia de aplicar las prácticas de fijación de precios de valor económico frente al valor del cliente en función de la configuración de creación de valor; por su parte, Hinterhuber (2017) menciona que en los mercados industriales las capacidades de cuantificación de valor siempre mejoran el rendimiento empresarial y es importante en el contexto de la fijación de precios basada en el valor, alinear los intereses del comprador y vendedor de la cadena de suministros.

En general, los estudios sobre negociación de precios se han centrado en el proceso de negociación de precios del proveedor, con una estructura de costos internos y percibiendo los precios como un parámetro de distribución de beneficios, en lugar de una oportunidad de colaboración con los clientes y de esta manera favorecer los beneficios industriales (Formentini y Romano, 2016). Sin embargo, las relaciones comerciales de colaboración dentro de la cadena de suministros ofrecen oportunidades para lograr un incremento de beneficio mutuo para las empresas, ese esfuerzo para mejorar la colaboración dentro de la cadena de suministro ya está extendido en cuanto a bienes y servicios (Voeth y Herbst, 2006). La gestión de negociación de precios colaborativos es más viable en empresas con relaciones establecidas por muchos años y surge de una experiencia colaborativa, de esta misma manera el desarrollo de los precios colaborativos permite a 
los clientes participar activamente en los precios de los productos (Chang y Yuan, 2008), donde el proceso interactivo de negociación de precios es una combinación de procesos colaborativos, predicción de necesidades, estimación de precios y mantenimiento de los beneficios.

Para avanzar en la comprensión sobre la negociación de precios en la cadena de suministros, se considera necesario realizar una investigación sobre los enfoques teóricos en las que se fundamentan los estudios que tratan los temas de negociación de precios. Como una alternativa surge el meta-análisis, un tipo de investigación científica que tiene como propósito integrar de forma objetiva y sistemática los resultados de los estudios empíricos sobre un determinado problema de investigación. La idea de muchos investigadores de combinar los resultados de estudios independientes para producir un conocimiento más amplio de un fenómeno de interés, es denominado método de trabajo de meta-análisis. Sin embargo, Ramirez-Correa y Garcia-Cruz (2005) consideraron conveniente denominar "enfoque meta-analítico" para diferenciarlo del metaanálisis cuantitativo. De esta manera, antes de emprender una investigación particular, el investigador debe preguntarse qué se sabe sobre el fenómeno, puesto que sin una visión clara sobre los últimos avances, un nuevo estudio que se realice no será de mucha utilidad. Sánchez-Meca (1999 citado en Ramirez-Correa y Garcia-Cruz, 2005) indica que las "revisiones de la investigación constituyen un eslabón imprescindible entre el quehacer científico del pasado y del futuro, estableciendo el punto de partida de nuevas investigaciones".

El incremento de las investigaciones es creciente y la identificación de los fundamentos teóricos más utilizados se convierte en una tarea indispensable, para la comprensión de las teorías utilizadas en la negociación de precios en la cadena de suministros, por lo cual se plantea el siguiente problema ¿Qué enfoques teóricos proponen o identifican los estudios realizados en los últimos años sobre el tema negociación de precios entre empresas de la cadena de suministros? Una vez delimitado el problema, el presente estudio tiene como objetivo general, identificar las teorías más utilizadas sobre el tema negociación de precios colaborativos entre empresas de la cadena de suministros en los estudios publicados durante los últimos cinco años, aplicando el enfoque meta analítico.

Formentini y Romano (2016) mencionan que las investigaciones sobre los precios colaborativos se realizaron solo con estudios conceptuales y matemáticos como la teoría de juegos, que no engloban completamente la actividad existente en la adopción y desarrollo de precios colaborativos. En este sentido el presente estudio contribuye proporcionando una visión general estructurada de las teorías más utilizadas en el tema de negociación de precios colaborativos entre empresas de la cadena de suministros. Para el logro del objetivo general, la metodología a aplicarse en la presente investigación tendrá una perspectiva exploratoria de tipo cualitativo a través del enfoque meta-analítico. A continuación se describe la metodología de revisión, luego se presenta y discute los resultados de la revisión de la literatura, sobre esta base en la cuarta sección se presenta las conclusiones finales de la investigación.

\section{METOdOLOGÍA}

La metodología tiene una perspectiva exploratoria por medio del enfoque meta-analítico. El enfoque metaanalítico aplica criterios para la selección de revistas artículos y autores; para elegir las revistas se seleccionaron aquellas con mayor factor de impacto. El factor de impacto mide la repercusión que ha tenido una revista en la literatura científica mediante el recuento de las citas que ha recibido. Si bien el factor de impacto tiene importantes limitaciones asociadas al sesgo de citas, el sesgo de cobertura y al cálculo mismo del factor, se reconoce que otros indicadores bibliométricos de valorización de calidad no han logrado la suficiente aceptación y uso generalizado para ser aplicados sistemáticamente (Aleixandre-benavent, Valderrama-Zurián y Golzález-Alcaide, 2007). Para elegir los artículos y autores se seleccionaron aquellos más citados, el enfoque meta-analítico también posibilita realizar un análisis de las técnicas estadísticas, técnicas muéstrales, líneas más investigadas, enfoques utilizados (Calazans, Paldes y Masson, 2016) análisis y exposición de resultados (Ramirez Correa y Garcia Cruz, 2005). Ramirez-Correa y Garcia-Cruz (2005) consideran que el enfoque meta analítico puede ser implementado en 4 fases; Por su parte, Melo-Mariano, et al. (2011 citado en Calazans et al., 2016) plantean 7 fases. Como se muestra en la figura 1, el método con enfoque meta-analítico utilizado para la revisión del fenómeno de estudio se puede describir en 6 fases.

Fase 1.- Determinar Revistas de la Disciplina

Fase 2.- Establecer Revistas relevantes

Fase 3.- Nutrir la Base de datos con artículos

Fase 4.- Análisis de autores y artículos

Fase 5.- Análisis de palabras claves

Fase 6.- Análisis y exposición de resultados

Fig. 1: Etapas del enfoque meta-analítico. 
La utilidad de la propuesta de enfoque meta-analítico de Ramírez-Correa y García-Cruz ( 2005) se puede constatar en trabajos como el de Pinzón, Vázquez, Elorza y Espejo (2010), desde la perspectiva metodológica, y en el de Grandón, Ramirez-Correa, y Rojas (2018), desde la perspectiva de resultados. Y la utilidad de la propuesta de Calazans et al. (2016) en el trabajo de Ayaviri y Saucedo-Estrada (2017).

La primera fase para explorar el dominio del problema es identificar cuáles son las revistas científicas utilizadas en el área estudiada, mediante una consulta en las bases de datos electrónicas, luego revisar las principales conferencias y congresos de asociaciones científicas que abordan el área en exploración, con el propósito de buscar artículos compilatorios de los trabajos presentados en ellas, donde el resultado de esta fase es un listado de revistas científicas utilizadas en el área estudiada, que servirán para establecer cuáles son las revistas más relevantes (fase 2) que serán la base del posterior análisis.

El análisis y revisión bibliográfica fue enfocado a la negociación de precios realizados entre las empresas, que se refiere a la transacción de productos y servicios en los niveles intermedios de la cadena de suministros, considerando una interacción entre comprador, proveedor y proveedores adicionales que pertenecen a la cadena de suministros. La selección de publicaciones relacionadas (fase 3) se realizó principalmente como una búsqueda de palabras clave estructuradas considerando la propuesta de Formentini y Romano (2016) que son las siguientes: pricing, pricing process, industrial pricing, B2B pricing, supply chain, collaboration, collaborative pricing, cooperative pricing, win-win y se complementó con collaborative negotiation, bargaining como palabras clave para consultar las bases de datos, con el fin de identificar las principales contribuciones relacionadas con los procesos de negociación de precios colaborativos entre las empresa de una cadena de suministro. La búsqueda en la base de datos de Web of Science se realizó en enero de 2019 para el periodo 2014 a 2018 con las palabras claves seleccionadas y combinadas con operadores lógicos de búsqueda para los campos de solo artículos, en las categorías de gestión, negocios e ingeniería industrial, en el área de negocios económicos, obteniéndose 7680 artículos. La fase 4,5 y 6 junto al análisis de datos fueron efectuados con la ayuda del Software Vosviewer, un programa informático de acceso libre desarrollado para construir y visualizar mapas bibliométricos.

\section{RESULTADOS Y DISCUSIÓN}

La cantidad de artículos identificados en 686 títulos de revistas muestran en la Figura 2 una línea de tendencia de crecimiento progresivo durante los últimos cinco años, en la que se observa el interés científico que va adquiriendo el tema que se estudia en el presente trabajo.

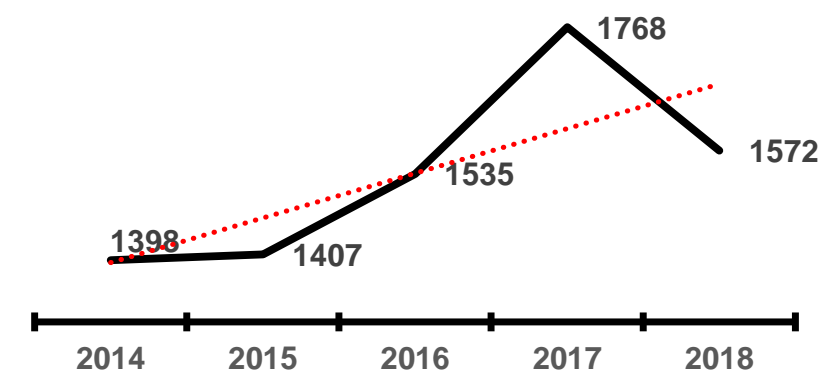

Fig. 2: Evolución Temporal de los Artículos Publicados (total 7680 artículos)

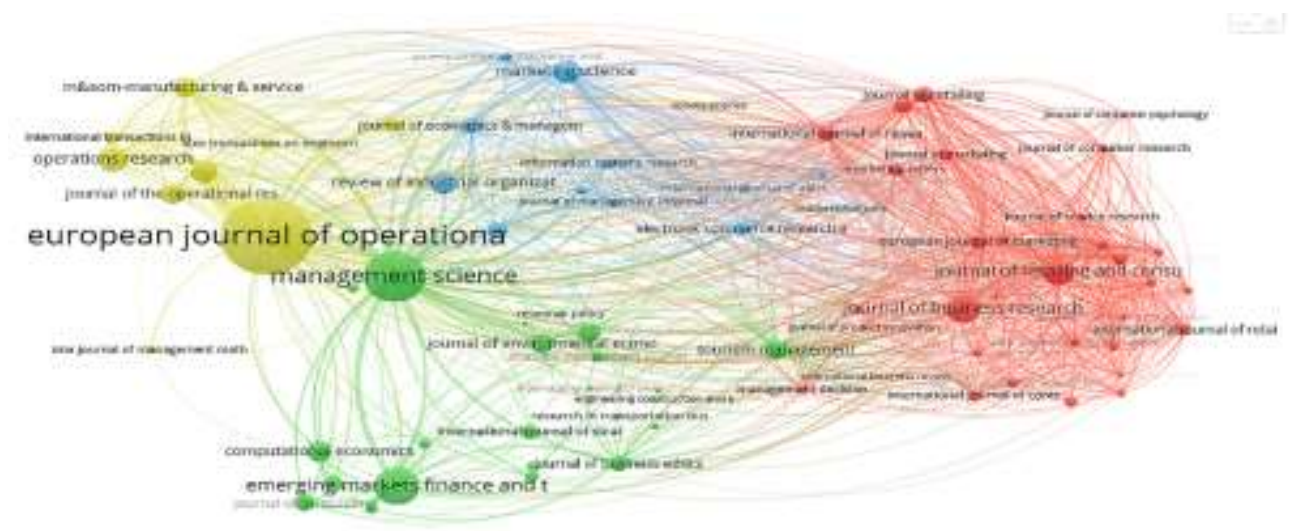

Fig. 3: Mapa de relaciones de revistas más citadas 
La Figura 3 muestra las revistas citadas más de 50 veces, entre las revistas con mayor número de citaciones y con más publicaciones se encuentran: European Journal of Operation Research y Management Science, que cuentan con el $33 \%$ de la publicaciones (953 artículos publicados), y con factor de impacto JCR mas alto están las revistas Journal of Marketing y Tourism Management como se puede observar en la Tabla 1.

Tabla1: Revistas más citadas de mayor relevancia según factor de impacto de JCR

\begin{tabular}{|c|c|c|c|}
\hline Título De Revista & Citaciones & Factor de Impacto & Artículos Publicados \\
\hline European Journal of Operational Research & 3737 & 3.428 & $21 \%$ \\
\hline Management Science & 2084 & 3.544 & $12 \%$ \\
\hline Tourism Management & 1054 & 5.927 & $3 \%$ \\
\hline Omega-International Journal of Management Science & 844 & 4.311 & $4 \%$ \\
\hline Journal of Business Research & 792 & 2.509 & $5 \%$ \\
\hline Journal of Retailing and Consumer Services & 771 & 2.919 & $5 \%$ \\
\hline Operations Research & 736 & 2.263 & $4 \%$ \\
\hline Technological Forecasting and Social Change & 624 & 3.131 & $3 \%$ \\
\hline Journal of Retailing & 604 & 5.48 & $2 \%$ \\
\hline Journal of Marketing Research & 560 & 3.854 & $2 \%$ \\
\hline Journal of Environmental Economics and Management & 553 & 2.635 & $3 \%$ \\
\hline Marketing Science & 515 & 2.794 & $4 \%$ \\
\hline International Journal of Forecasting & 447 & 2.186 & $2 \%$ \\
\hline Journal of Marketing & 393 & 7.338 & $2 \%$ \\
\hline Mysom-Manufacturing y Service Operations Management & 384 & 1.795 & $3 \%$ \\
\hline Emerging Markets Finance and Trade & 305 & 0.828 & $8 \%$ \\
\hline International Journal of Research in Marketing & 294 & 2.593 & $2 \%$ \\
\hline Journal of Business Ethics & 286 & 2.917 & $2 \%$ \\
\hline Journal of Consumer Research & 224 & 3.535 & $1 \%$ \\
\hline International Journal of Contemporary Hospitality Management & 218 & 2.874 & $1 \%$ \\
\hline Information Systems Research & 218 & 2.301 & $1 \%$ \\
\hline Review of Industrial Organization & 214 & 0.767 & $3 \%$ \\
\hline Computational Economics & 209 & 1.038 & $3 \%$ \\
\hline Research Policy & 198 & 4.661 & $1 \%$ \\
\hline Journal of the Operational Research Society & 197 & 1.396 & $3 \%$ \\
\hline
\end{tabular}

Tabla 2: Autores más citados y con mayor número de publicaciones

\begin{tabular}{llcc}
\hline \multicolumn{1}{c}{ Autor } & \multicolumn{1}{c}{ Institución } & Citaciones & Artículos \\
\hline Cheng Tai Chiu, Edwin & $\begin{array}{l}\text { Escuela de Ciencias de la Administración e } \\
\text { Ingeniería, Universidad de Nanjing, China }\end{array}$ & 130 & 19 \\
\hline Choi, Tsan-Ming & $\begin{array}{l}\text { División de Negocios, Instituto de Textiles y Ropa, } \\
\text { Universidad Politécnica de Hong Kong, Hong Kong }\end{array}$ & 128 & 12 \\
\hline Li, Yongjian & Escuela de Negocios, Universidad de Nankai, China & 126 & 7 \\
\hline Chen, Xu & $\begin{array}{l}\text { Escuela de Administración y Economía, Universidad } \\
\text { de Ciencia y Tecnología Electrónica de China, China }\end{array}$ & 123 & 6 \\
\hline Govindan, Kannan & $\begin{array}{l}\text { Departamento de Economía y Negocios, } \\
\text { Universidad del Sur de Dinamarca, Dinamarca }\end{array}$ & 118 & 5 \\
\hline Wang, Xiaojun & $\begin{array}{l}\text { Escuela de Economía, Finanzas y Gestión, } \\
\text { Universidad de Bristol, Reino Unido }\end{array}$ & 114 & 7 \\
\hline Gendreau, Michel & Escuela Politecnica de CIRRELT y MAGl, Canada & 103 & 103 \\
\hline Pauwels, Koen & Universidad Ozyegin de Estambul, Turquía & 9 \\
\hline
\end{tabular}

El análisis efectuado con el Software Vosviewer muestra que Cheng Tai Chiu, Edwin y Choi, Tsan-Ming son los autores más citados con mayor número de publicaciones realizadas en el periodo de estudio, estos autores debieran considerarse en un proceso de revisión de la literatura sobre el tema de investigación (ver Tabla 2). Por otra parte, en la Figura 4 se puede observar los artículos más citados, donde (Belleflamme, Lambert, y Schwienbacher (2014) con el artículo titulado "Crowdfunding: Tapping the right crowd" es el más citado, 
también se mencionan publicaciones de Cachon (2014), Zhang, Wang, y You (2015) y Cornelissen y Werner (2014) como las más relevantes sobre el tema de estudio.

De las revistas de mayor impacto JCR se seleccionaron los artículos publicados que fueron citados más de 50 veces, identificándose 16 documentos que se presentan en la Tabla 3 y complementan a los documentos que se muestran en la Figura 4. En el análisis de palabras claves se organiza los artículos por palabras clave, con la finalidad de detectar los tópicos más importantes que se están estudiando en el campo de la investigación. Como indica Uddin y Khan (2016) las palabras clave son muy importantes para asegurar que los lectores estén conscientes de los artículos científicos y de su contenido. De la misma manera Khan y Wood (2015) manifiestan que las palabras clave proporcionadas por el autor son las que mejor representan el tema de investigación que se discute en los documentos.

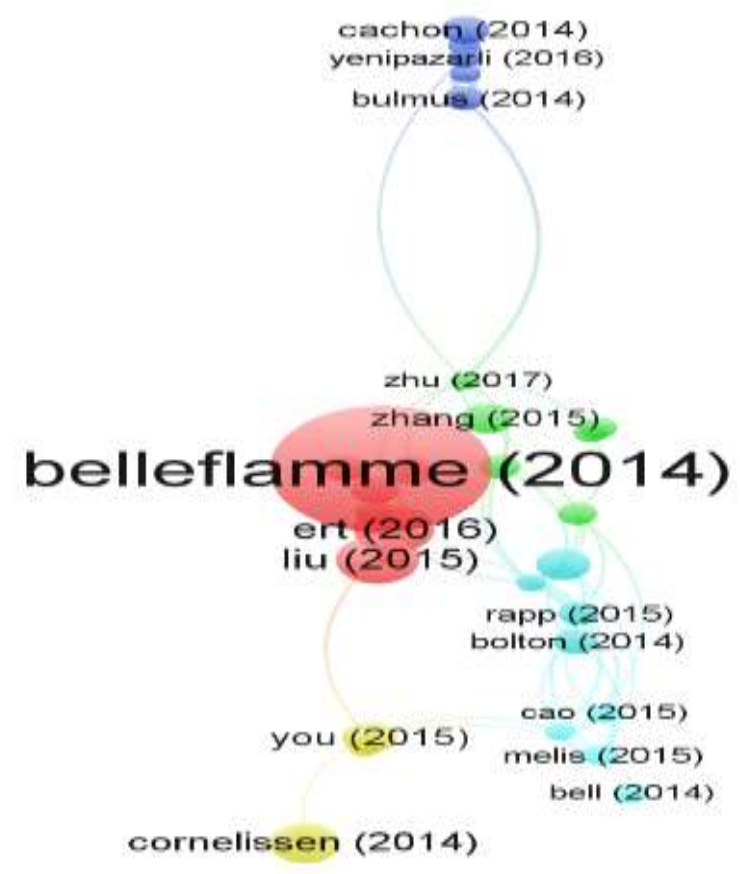

Fig. 4: Artículos más citados y sus relaciones

La Figura 5 muestra el mapa de relaciones de palabras claves distribuidos en tres grupos, donde las más relacionadas son: Model, Price, Information, Competition, Pricing, Management,Supply Chain, Game Theory, Market, Models, Industry.

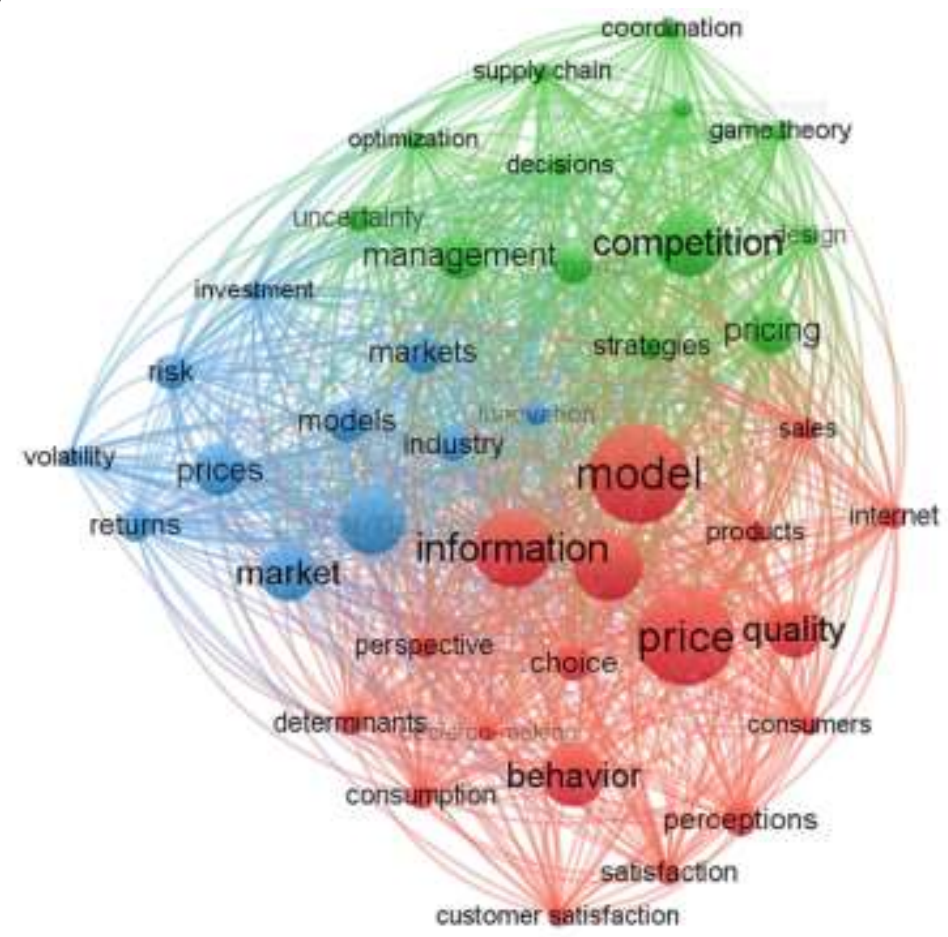

Fig. 5: Mapa de relaciones entre palabras claves de artículos analizados 
Tabla 3: Artículos más citados en revistas de mejor ranking SJR

\begin{tabular}{|c|c|c|c|c|}
\hline Autor & Titulo & Revista & Año & Citaciones \\
\hline Liu, ZW; Park, S & $\begin{array}{l}\text { What makes a useful online } \\
\text { review? Implication for } \\
\text { travel product websites }\end{array}$ & $\begin{array}{l}\text { Tourism } \\
\text { Management }\end{array}$ & 2015 & 113 \\
\hline Ert, E; Fleischer, A; Magen, N & $\begin{array}{l}\text { Trust and reputation in the } \\
\text { sharing economy: The role } \\
\text { of personal photos in Airbnb }\end{array}$ & $\begin{array}{l}\text { Tourism } \\
\text { Management }\end{array}$ & 2016 & 109 \\
\hline $\begin{array}{l}\text { Hong, T; Pinson, P; Fan, S; Zareipour, H; } \\
\text { Troccoli, A; Hyndman, RJ }\end{array}$ & $\begin{array}{l}\text { Probabilistic energy } \\
\text { forecasting: Global Energy } \\
\text { Forecasting Competition } \\
2014 \text { and beyond }\end{array}$ & $\begin{array}{l}\text { International } \\
\text { Journal Of } \\
\text { Forecasting }\end{array}$ & 2016 & 101 \\
\hline Han, H; Hyun, SS & $\begin{array}{l}\text { Customer retention in the } \\
\text { medical tourism industry: } \\
\text { Impact of quality, } \\
\text { satisfaction, trust, and price } \\
\text { reasonableness }\end{array}$ & $\begin{array}{l}\text { Tourism } \\
\text { Management }\end{array}$ & 2015 & 74 \\
\hline You, Y; Vadakkepatt, GG; Joshi, AM & $\begin{array}{l}\text { A Meta-Analysis of } \\
\text { Electronic Word-of-Mouth } \\
\text { Elasticity }\end{array}$ & $\begin{array}{l}\text { Journal Of } \\
\text { Marketing }\end{array}$ & 2015 & 73 \\
\hline Zhang, LH; Wang, JG; You, JX & $\begin{array}{l}\text { Consumer environmental } \\
\text { awareness and channel } \\
\text { coordination with two } \\
\text { substitutable products }\end{array}$ & $\begin{array}{l}\text { European Journal } \\
\text { Of Operational } \\
\text { Research }\end{array}$ & 2015 & 71 \\
\hline Rossi, PE & $\begin{array}{l}\text { Even the Rich Can Make } \\
\text { Themselves Poor: A Critical } \\
\text { Examination of IV Methods } \\
\text { in Marketing Applications }\end{array}$ & Marketing Science & 2014 & 66 \\
\hline Cachon, GP & $\begin{array}{l}\text { Retail Store Density and the } \\
\text { Cost of Greenhouse Gas } \\
\text { Emissions }\end{array}$ & $\begin{array}{l}\text { Management } \\
\text { Science }\end{array}$ & 2014 & 64 \\
\hline Zervas, G; Proserpio, D; Byers, JW & $\begin{array}{l}\text { The Rise of the Sharing } \\
\text { Economy: Estimating the } \\
\text { Impact of Airbnb on the } \\
\text { Hotel Industry }\end{array}$ & $\begin{array}{l}\text { Journal Of } \\
\text { Marketing } \\
\text { Research }\end{array}$ & 2017 & 63 \\
\hline
\end{tabular}

Kriegler, E; Riahi, K; Bauer, N; Schwanitz, VJ;

Petermann, N; Bosetti, V; Marcucci, A; Otto, S;

Paroussos, L; Rao, S; Curras, TA; Ashina, S;

Bollen, J; Eom, J; Hamdi-Cherif, M; Longden, T;

Kitous, A; Mejean, A; Sano, F; Schaeffer, M;

Wada, K; Capros, P; van Vuuren, DP;

Edenhofer, O

Singleton, KJ

Sexton, SE; Sexton, AL

Tang, CF; Tan, EC

Rapp, A; Baker, TL; Bachrach, DG; Ogilvie, J; Beitelspacher, LS

Karger, DR; Oh, S; Shah, D

Xiao, TJ; Choi, TM; Cheng, TCE
Making or breaking climate targets: The AMPERE study on staged accession scenarios for climate policy

Technological

Forecasting And 2015

62

Social Change

\begin{tabular}{|c|c|c|c|}
\hline $\begin{array}{l}\text { Investor Flows and the } \\
2008 \text { Boom/Bust in Oil } \\
\text { Prices }\end{array}$ & $\begin{array}{l}\text { Management } \\
\text { Science }\end{array}$ & 2014 & 59 \\
\hline $\begin{array}{l}\text { Conspicuous conservation: } \\
\text { The Prius halo and } \\
\text { willingness to pay for } \\
\text { environmental bona fides }\end{array}$ & $\begin{array}{l}\text { Journal Of } \\
\text { Environmental } \\
\text { Economics And } \\
\text { Management }\end{array}$ & 2014 & 58 \\
\hline $\begin{array}{l}\text { Does tourism effectively } \\
\text { stimulate Malaysia's } \\
\text { economic growth? }\end{array}$ & $\begin{array}{l}\text { Tourism } \\
\text { Management }\end{array}$ & 2015 & 57 \\
\hline $\begin{array}{l}\text { Perceived customer } \\
\text { showrooming behavior and } \\
\text { the effect on retail } \\
\text { salesperson self-efficacy } \\
\text { and performance }\end{array}$ & $\begin{array}{l}\text { Journal Of } \\
\text { Retailing }\end{array}$ & 2015 & 55 \\
\hline $\begin{array}{l}\text { Budget-Optimal Task } \\
\text { Allocation for Reliable } \\
\text { Crowdsourcing Systems }\end{array}$ & $\begin{array}{l}\text { Operations } \\
\text { Research }\end{array}$ & 2014 & 53 \\
\hline $\begin{array}{l}\text { Product variety and channel } \\
\text { structure strategy for a } \\
\text { retailer-Stackelberg supply } \\
\text { chain }\end{array}$ & $\begin{array}{l}\text { European Journal } \\
\text { Of Operational } \\
\text { Research }\end{array}$ & 2014 & 51 \\
\hline
\end{tabular}




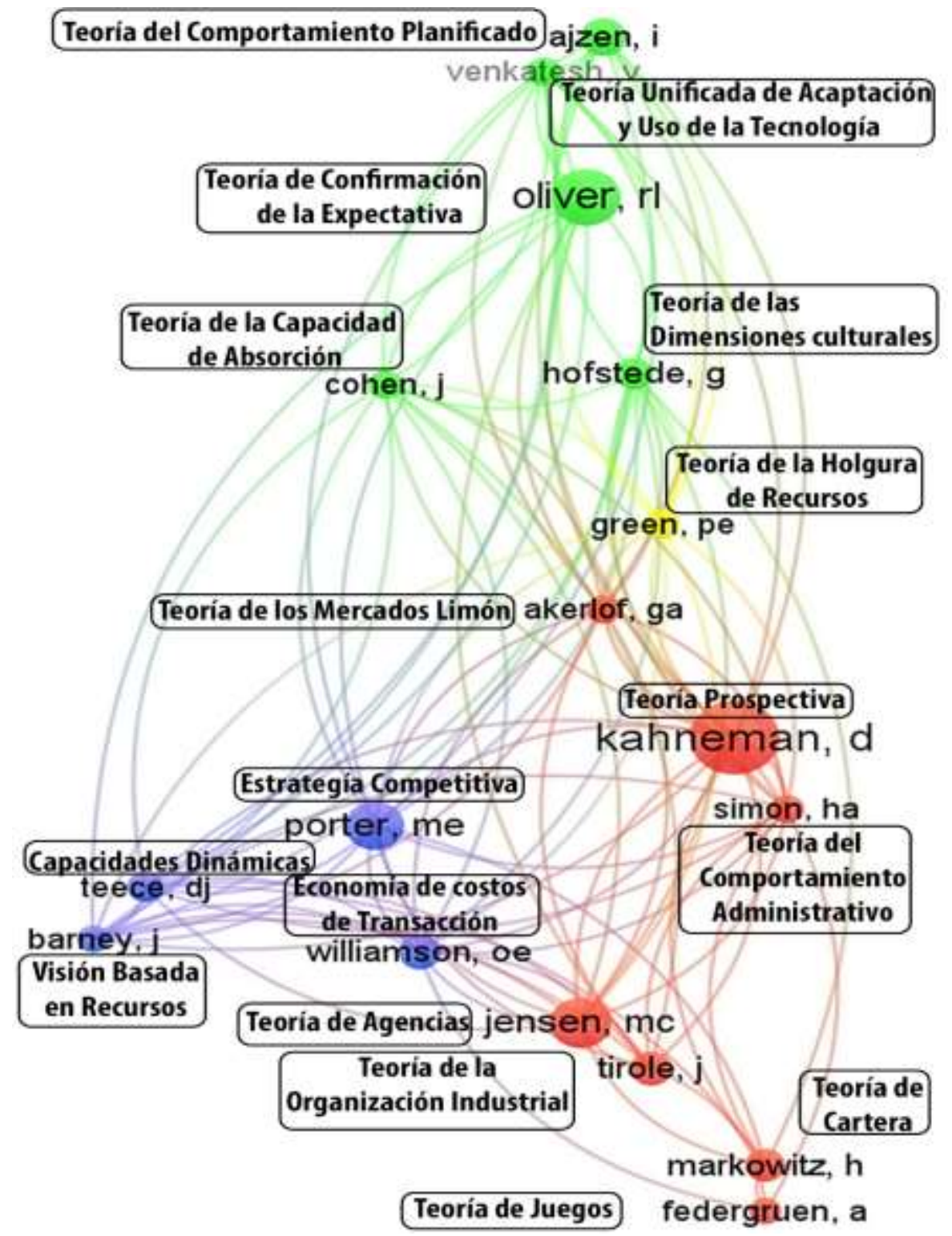

Fig. 6: Mapa de relaciones de autores y enfoques teóricos más citados en los artículos analizados

En la etapa seis se realiza el análisis y la exposición de resultados, en nuestro caso es importante investigar cuales son los enfoques que más abordaron y el aporte realizado entre otras informaciones obtenidas de los artículos seleccionados. Para identificar las principales teorías referidas en los documentos analizados, se realizó un análisis de co-citación con el software Vosviewer seleccionando a los autores citados más de 100 veces; por otra parte, se seleccionó la relación de autores principales y enfoques teóricos de los documentos de Dwivedi, Wade y Schneberger, (2011); Miranda-Torrez, (2017) y Formentini y Romano, (2016), y se construyó la relación de autores con los enfoques teóricos más referenciados en la literatura relacionada con el tema de estudio expuestos en la Figura 6 y complementando la información en la Tabla 4, donde se muestra la relación de teorías que fueron citadas más de 100 veces agrupadas en cuatro grupos. El primer grupo de color rojo agrupa siete teorías que están relacionadas con las teorias de capacidad organizacional en condiciones de riesgo, Formentini y Romano (2016) manifiesta la aplicación de la teoria de juegos para una distribucion equitativa de ganancias y el riego relacionado con la negociacion de precios que recuerda a la teoria de agencias. El grupo dos de color verde asocia principalmente teorías relacionadas con el comportamiento del mercado, el tercer grupo de color azul agrupa a las teorías de enfoque estratégico y ventaja competitiva (Miranda-Torrez, 2017) y un cuarto grupo que considera a la teoría de la Holgura de Recursos.

Las teorías identificadas en el presente estudio se respaldan en gran medida con los estudios de Sanderson, Lonsdale, Mannion y Matharu (2015), Formentini y Romano (2016), Ribbink y Grimm (2014) y Cao, 
Vonderembse, Zhang y Ragu-Nathan (2010). Sanderson et al. (2015) en su revisión de la literatura, realizo una categorización en base a los procesos desarrollados en la adquisición y gestión de cadena de suministros y asocian con la Teoría de Agencia, Teoría de Economía de Costos de Transacción, Teoría de las Capacidades Dinámicas y Teoría de Juegos. Por su parte, Formentini y Romano (2016) menciona que los principales estudios revisados sugieren la aplicación de la Teoría de Juegos, la Teoría de Agencia y la Economía de Costos de Transacción. Ribbink y Grimm (2014) desarrollaron un modelo para abordar el impacto de la cultura en una relación comprador-proveedor internacional con base en la teoría de la Visión Relacional y la Economía de los Costos de Transacción. Cao et al. (2010) respaldado en la Teoría de Recursos y Capacidades y la Economía de Costos de Transacción, manifiesta que las empresas luchan por una mayor colaboración en la cadena de suministros, aprovechando los recursos y el conocimiento de proveedores clave para reducir la incertidumbre, reducir los costos de transacción, capitalizar oportunidades de aprendizaje para mejorar la posición competitiva. Por lo cual las teorías identificadas podrían desempeñar un papel importante al gestionar la negociación de precios colaborativos como una forma para crear ventaja competitiva entre empresas de la cadena de suministros.

Tabla 4: Enfoques Teóricos más Citados

\begin{tabular}{clll}
\hline Grupo & \multicolumn{1}{c}{ Teorías } & \multicolumn{1}{c}{ Autor } & Citaciones \\
\hline \multirow{4}{*}{1} & La Teoría Prospectiva & Kahneman, Daniel & 520 \\
\cline { 2 - 4 } & Teoría de Agencia & Jensen, Michael Cole & 275 \\
\cline { 2 - 4 } & Teoría de la Organización Industrial & Tirole, Jean & 156 \\
\cline { 2 - 4 } & Teoría de Cartera & Markowitz, Harry & 135 \\
\cline { 2 - 4 } & Teoría de los Mercados Limón. & Akerlof,George Arthur & 110 \\
\cline { 2 - 4 } & Teoría del Comportamiento Administrativo & Simon, Herbert Alexander & 110 \\
\cline { 2 - 4 } & Teoría de Juegos & Federgruen, Awi & 103 \\
\hline \multirow{3}{*}{2} & Teoría de la Confirmación de la Expectativa & Oliver, Richard L. & 339 \\
\cline { 2 - 4 } & Teoría del Comportamiento Planificado & Ajzen, Icek & 169 \\
\cline { 2 - 4 } & Teoría de las Dimensiones Culturales & Hofstede, Gerard Hendrik & 131 \\
\cline { 2 - 4 } & Teoría de la Capacidad de Absorción & Cohen, Jacob & 111 \\
\cline { 2 - 4 } & Teoría Unificada de Aceptación y Uso de la Tecnología & Venkatesh, Viswanath & 111 \\
\hline \multirow{3}{*}{3} & Estrategia Competitiva & Porter, Michael Eugene & 262 \\
\cline { 2 - 4 } & Economía de Costos de Transacción & Williamson, Oliver Eaton & 153 \\
\cline { 2 - 4 } & Capacidades Dinámicas & Teece, David John & 119 \\
\cline { 2 - 4 } & Recursos y capacidades & Barney, Jay & 103 \\
\hline 4 & Teoría de la Holgura de Recursos & Green, Paul E. & 119 \\
\hline
\end{tabular}

\section{CONCLUSIONES}

Se tiene una ausencia de revisión de literatura con respecto a la colaboración de la cadena de suministros en el fenómeno del proceso de fijación de precios a través de teorías establecidas (Formentini y Romano, 2016), en este sentido, el presente estudio proporciona una contribución teórica al creciente interés científico que se tiene sobre negociación de precios colaborativos entre empresas de la cadena de suministros. Se identificaron cuatro grupos de teorías en el ámbito de estudio: las relacionadas con la capacidad organizacional en condiciones de riesgo, el comportamiento del mercado, el enfoque estratégico competitivo y los recursos. Destacandose por su citación la teoria prospectiva de (Kahneman y Tversky, 1979) y la teoria de la confirmacion de la expectativa (Oliver y Burke, 1999). Adicionalmente, las teorías de recursos y capacidades de Barney, costos de transaccion (Williamson, 2010), estrategia competitiva de Porter M.E. y el comportamiento planificado de Ajzen, se consideran relevantes para el estudio del tema de negociación de precios colaborativos entre empresas de las cadenas de suministro, evaluando la oportunidad de precibir los precios colaborativos como una forma de crear ventaja competitiva (Formentini y Romano, 2016).

\section{REFERENCIAS}

Aleixandre-Benavent, R., J. C. Valderrama-Zurián, G. Golzález-Alcaide, El Factor de Impacto de las Revistas Científicas: Limitaciones en lindicadores Alternativos, El Profesional de la Información, 16(1), 4-11 (2007)

Ayaviri, A. y H. K. Saucedo-Estrada, Supply Chain Management: A Review of the Literature Applying the Meta-Analytical Approach, Ecorfan Journal, 3(5), 43-59 (2017)

Belleflamme, P., T. Lambert y A. Schwienbacher, Crowdfunding: Tapping the right crowd, Journal of Business Venturing, 29(5), 585-609 (2014)

Cachon, G. P., Retail Store Density and the Cost of Greenhouse Gas Emissions, Management Science, 60(8), 1907-1925 (2014)

Calazans, A. T., R.A. Paldes y E. T. Masson, Uma revisão sistemática da bibliografia sobre usabilidade móvel utilizando o enfoque meta-analítico, Espacios, 37(No 10), 1-11 (2016) 
Cao, M., M. Vonderembse, Q. Zhang y T. S. Ragu-Nathan, Supply Chain Collaboration: Conceptualisation and Instrument Development, doi:10.1080/00207540903349039, International J. of Production Research, 48(22), 6613-6635 (2010)

Chang, W. y S.T. Yuan, Collaborative Pricing Model for Bundling Information Goods, doi:10.1177/0165551507084632, Journal of Information Science, 34(151), 635-650 (2008)

Cornelissen, J. P. y M.D. Werner, Putting Framing in Perspective : A Review of Framing and Frame Analysis across the Management and Organizational Literature, doi: 10.1080/19416520.2014.875669, Academy of Management Annals, 8(1), 181-235, (2014)

Dwivedi, Y. K., M.R. Wade y S.L. Schneberger, Information Systems Theory: Explaining and Predicting Our Digital Society,

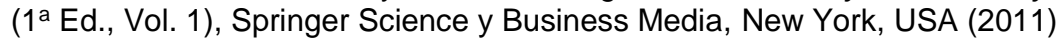

Formentini, M. y P. Romano, Towards Supply Chain Collaboration in B2B Pricing: A Critical Literature Review and Research Agenda, doi:10.1108/IJOPM-03-2015-0124, International Journal Of Operation And Production Management, 36(7), 734-756 (2016)

Grandón, E. E, P.E. Ramirez-Correa y K. P. Rojas, Uso de la teoría business process change (bpc) para examinar la adopción de enterprise resource planning (erp) en Chile, Interciencia, 43(10), 716-722 (2018)

Hinterhuber, A., Value Quantification Capabilities in Industrial Markets, doi: 10.1016/j.jbusres.2016.11.019, Journal of Business Research, 76, 163-178 (2017)

Johansson, M. y L. Andersson, Pricing Practices and Value Creation Logics, doi: 10.1057/rpm.2011.43, Journal of Revenue and Pricing Management, 11(1), 64-75(2012)

Kahneman, D. y A. Tversky, Prospect Theory: An Analysis of Decision under Risk, Econometrica, 47(2), 263-292 (1979)

Khan, G. F. y J. Wood, Information Technology Management Domain: Emerging Themes and Keyword Analysis, doi:10.1007/s11192-015-1712-5, Scientometrics (2015)

La Londe, B. J. y J.M. Masters, Emerging Logistics Strategies: Blueprints for the Next Century, Internaitonal Journal of Physical Distribution and Logistics Management, 24(7), 35-47 (1994)

Mentzer, J. J. T., W. Dewitt y otros cinco autores, Defining Supply Chain Management, doi:10.1002/j.21581592.2001.tb00001.x, Journal of Business Logistics, 22(2), 1-25 (2001)

Miranda-Torrez, J., Las Teorías y la Conceptualización de las Capacidades Dinámicas Estratégicas y Organizacionales, XXII Congreso Internacional de Contaduría, Administración e Informática, (2017)

Oliver, R. L. y R.R. Burke, Expectation Processes in Satisfaction Formation A Field Study, Journal of Service Research, 1(3), 196-214 (1999)

Pinzón, P. A., J. Vázquez, M. V. Elorza y M.A. D. Espejo, Internacionalización y Control Interorganizativo: Revisión Teórica y Propuestas de Investigación, doi: 10.1016/S1135-2523(12)60008-2, Investigaciones Europeas de Dirección y Economía de La Empresa, 16(1), 165-180 (2010)

Ramirez-Correa, P. y R. Garcia-Cruz, Meta-análisis sobre la Implantacion de Sistemas de Planificación de Recursos Empresariales (ERP), Revista de Gestao de Tecnologia e Sistemas de Inforamacao, 2(3), 245-273 (2005)

Ribbink, D. y C. M. Grimm, The Impact of Cultural Differences on Buyer-Supplier Negotiations: An Experimental Study, doi: 10.1016/j.jom.2014.01.004, Journal of Operations Management, 32(3), 114-126, (2014)

Salas-Navarro, K., J.A. Meza, T. Obredor-Baldovino y N. Mercado-Caruso, Evaluación de la Cadena de Suministro para Mejorar la Competitividad y Productividad en el Sector Metalmecánico en Barranquilla, Colombia, Información Tecnológica, 30(2), 25-32 (2019)

Sanchis, R., R. Poler y A. Ortiz, Técnicas para el Modelado de Procesos de Negocio de Cadenas de Suministro, doi: 10.1612/inf.tecnol.4017it.08,Información Tecnológica, 20(2), 29-40.,(2009)

Sanderson, J., C. Lonsdale, R. Mannion y T. Matharu, Towards a Framework for Enhancing Procurement and Supply Chain Management Practice in the NHS: Lessons for Managers and Clinicians from a Synthesis of the Theoretical and Empirical Literature, (Vol. 3), National Institute for Helath Research (2015)

Sarkis, J., Q. Zhu y K. Lai, An Organizational Theoretic Review of Green Supply Chain Management Literature, doi: 10.1016/j.jpe.2010.11.010, International Journal of Production Economics, 130(1), 1-15 (2011)

Uddin, S. y A. Khan, The Impact of Author-Selected Keywords on Citation Counts, doi:10.1016/j.joi.2016.10.004,Journal of Informetrics, 10(4), 1166-1177 (2016)

Voeth, M. y U. Herbst, Supply Chain Pricing - A New Perspective on Pricing in Industrial Markets, doi:10.1016/j.indmarman.2005.08.010, Industrial Marketing Management, 35, 83-90 (2006)

Williamson, O. E., Transaction Cost Economics: The Natural Progression, doi:10.1016/j.jretai.2010.07.005, Journal of Retailing, 86(3), 215-226 (2010)

Zhang, L., J. Wang y J. You, Consumer Environmental Awareness and Channel Coordination with Two Substitutable Products, doi:10.1016/j.ejor.2014.07.043,European Journal of Operational Research, 241(1), 63-73 (2015) 